\title{
Hand Hygiene: A Quality Improvement Project
}

\author{
Abigail Mitchell*1,Emma Boisvert ${ }^{2}$, Tiffany Wilson ${ }^{3}$ and Shannon Hogan ${ }^{4}$ \\ ${ }^{1}$ DHEd, MSN, RN, CNE, FHERDSA \\ ${ }^{2}$ Emma Boisvert RN, BSN \\ ${ }^{3}$ Tiffany Wilson RN \\ ${ }^{4}$ Shannon Hogan RN, BSN,CCRN
}

Received: December 12, 2017; Published: December 18, 2017

*Corresponding author: AAbigail Mitchell, Professor, DHEd MSN, RN, CNE, FHERDSA; Email: mitchela@dyc.edu

\begin{abstract}
Health care associated infections (HAIs) are known world-wide primarily due to poor hand hygiene practices. It is imperative for family nurse practitioners (FNPs) to be role models in the workplace exemplifying proper hand hygiene techniques and advocating for patient safety. FNPs can do this by educating those who do not perform or are not knowledgeable of proper hand hygiene practices. The Centers for Disease Control and Prevention (CDC) [1] recommend that hand hygiene consist of decontaminating hands with antiseptics routinely, when hands are soiled, before and after any patient interaction and prior to health-care related procedures. Florence Nightingale established the environmental theory in the 1800 's, as she began to recognize the importance in maintaining a clear environment to best promote the health of her patients.

Abbreviations: WHO: World Health Organization; HAI: Health Care Associated Infections; NIH: National Institute of Health; CDC: Center for Disease Control; HICPAC: Healthcare Infection Control Practices Advisory Committee; NP: Nurse Practitioners; RN: Registered Nurses; CNA: Certified Nursing Assistants
\end{abstract}

\section{Research Question}

When provided with educational materials on hand hygiene, is there an increase in knowledge among healthcare professionals in acute care settings?

\section{Problem Statement}

According to the World Health Organization (WHO), health care associated infections (HAI) cause major problems for patient safety and health promotion [2]. The impacts of HAIs include prolonged hospital stays, long-term disabilities, increased resistance of microorganisms to antimicrobial treatments, financial burdens, high costs for patient care that is not reimbursable by insurance agencies and mortality [2]. The risk of patients developing HAIs is universal and exists in every healthcare facility and system worldwide [2]. Studies estimate that 1.4 million patients throughout the world are affected at any one time with an HAI [2]. In the United States (U.S.) alone, Haverstick et al. [3] reports on research that revealed about 1 in 25 patients in the acute-care setting will develop a HAI during their stay. Data from 2011 shows that about 700,000 patients had an HAI in the U.S. and $10 \%$ of those patients died from complications related to the HAIs. There have been many studies completed by numerous organizations, to include the National Institute of Health (NIH) [4], showing that the lack of hand hygiene among healthcare professionals contributes to the most HAIs in patients [2]. Prior studies have shown that hand hygiene compliance can be hindered by unsafe patient to nurse ratios, skin irritation from the antimicrobial treatment, and lack of knowledge. The persistence of staff education regarding practices can be a critical measure in reducing HAIs [5]. Therefore, the purpose of this study is to provide educational materials to healthcare professionals on acute care units in hospitals. Consequently, this will result in increased knowledge on the importance of hand hygiene and the proper technique of hand hygiene to reduce HAIs.

\section{Florence Nightingale}

Florence Nightingale is one of the most recognized names in nursing. Nightingale was born in 1820 in Italy to a wealthy British family, who, in 1844, were angry when she told them her decision of becoming a nurse. Nightingale became known for her work in the field during the Crimean War. She became known at the "Lady with the Lamp," because of her night rounds tending to wounded soldiers [6]. Nightingale started a Sanitary Commission after she pointed out that the unsanitary conditions of the soldiers were a major cause of death. Her work led to reduced death rates from $42 \%$ to $2 \%$ [6]. Hand hygiene compliance in health care professionals today is important in preventing the spread of infections.

\section{Literature Review}

A comprehensive literature review was conducted through multiple databases and search engines including Google Scholar, EBSCO Host, and CINAHL nursing database. The inclusive criteria 
for research collection included articles between 2012 and 2017 Search terms included "hand washing, prevention of illness, flu season, and hand washing compliance." With infections today adapting and becoming stronger, the need for protection in the hospital setting is at an all-time high. Hand hygiene is an easy and very efficient way of preventing the spread of infections.

\section{Hand Hygiene: Past to Present}

Ignaz Semmelweis was a Hungarian physician of ethnic-German ancestry, known as the "father of hand hygiene". Semmelweis along with other colleagues established that hospital acquired infections were transmitted through the hands during activities with patients [2]. In 1847, Semmelweis discovered this after witnessing an alarmingly high number of mortality rates from puerperal fever at an obstetric clinic in which he held the position house officer. Semmelweis proposed all healthcare professionals wash their hands with a chlorinated lime solution before all patient contact and as a result the mortality rate at the obstetrics clinic fell to dramatically to three percent and subsequently remained low [2]. Since Semmelweis initial hand hygiene implementation, hundreds of studies and investigations have been conducted to provide evidence based practice recommendations on hand hygiene and the prevention of hospital acquired infections.

In the 1980's the first national guidelines for hand hygiene were published and in 1995 and 1996, the Center for Disease Control (CDC)/Healthcare Infection Control Practices Advisory Committee (HICPAC) recommended either antimicrobial soap or a waterless antiseptic agent to be used for cleansing hands for all healthcare professionals entering and exiting patient rooms to decrease the spread of multidrug-resistant pathogens [2]. In 2002, HICPAC released guidelines that define alcohol-based hand rubbing, as the standard of care for hand hygiene practices in healthcare settings and that hand washing is reserved for specific situations only. The current CDC recommendations for hand hygiene in healthcare settings include decontaminating hands with healthcare facility approved antiseptics when hands are visibly soiled, routinely, before direct contact with a patient, before donning gloves and after removing gloves and when performing any healthcare related procedure on a patient.

\section{Hand Hygiene Barriers}

Although, 100\% hand hygiene compliance may seem like a straightforward and effortless task, a variety of challenges have been recognized as hindrances to accomplishing this objective. WHO [2] presented common barriers of hand hygiene including "skin irritation caused by hand hygiene agents, inaccessible hand hygiene supplies, interference with HCW [health care worker]patient relationships, patient needs perceived as a priority over hand hygiene, wearing of gloves, forgetfulness, lack of knowledge of guidelines, insufficient time for hand hygiene, high workload and understaffing, among other issues. Kirk et al. [7] performed a crosssectional examination of health care workers utilizing a survey to inquire about one's knowledge, attitude, and self-reported practice of point of care hand hygiene. A convenience sample of 200 health care providers in the United States and 150 health care providers in Canada were chosen for the study. Half of the respondents were physicians and half were nurses. Forty-one percent of the respondents listed "dispensers/sinks not in a convenient location", $36 \%$ reported "being busy", and 32\% reported "products dry our hands" as barriers. Increased workload and crowding was recognized as a main factor to low hand hygiene compliance in an observational study performed over 22- months in a 40-bed emergency department located in Toronto, Ontario, Canada [8].

Although this study is limited to a single emergency department with potential bias of an observational study, the theme of increased workload as a barrier to hand hygiene is valid. In a society involving technology in all facets of the health care system, it's very important to comment on the use of mobile phones and the potential barrier to proper hand hygiene. A study performed by Mark et al. [9], at a hospital in Northern Ireland, involved swabbing 50 mobile phones for bacterial growth and simultaneously administering a questionnaire investigating cell phone usage among staff. Sixty percent of the phones yielded bacterial growth. The results from the questionnaire found $45 \%$ of the participants never wash their hands after cell phone usage and $63 \%$ report never decontaminating their phone. In addition, 57\% stated that if their phone was proven to be contaminated, this would change their hand hygiene practice when using their device [9]. Despite these barriers, is it possible to educate hospital staff about the overwhelming importance of proper hand hygiene to result in increased hand hygiene compliance?

\section{Methodology}

A 13-question Quality Improvement survey was developed to assess healthcare professionals' baseline knowledge regarding hand hygiene practices. The survey included 10 knowledge questions and three demographic questions. This tool was developed using various resources including: National Institute of Health (NIH) [4], Healthy People 2020, CDC and WHO. This survey was used as a pre-test to assess the base knowledge of healthcare personnel. These surveys were sent via email to nurses, physicians, mid-level providers, and nursing assistants. These staff members work on medical surgical units and/or intensive care units. The research took place in three different locations: Bennington, Vermont, Norfolk, Virginia, and Washington D.C./Maryland. After obtaining permission from each nurse manager from each region, an email was sent to approximately 75 people at each location [10]. This email contained information on the quality improvement project, a consent letter, a link to the survey tool, and contact information of the researchers. Finally, the participants were asked to complete this pre-test in anticipation of receiving an educational PDF file (poster) and post-test to be sent out in the near future.

An email containing a one-page educational PDF (poster) attachment and a link to the post-test was emailed to the same sample population. The educational PDF file (poster) contained brief sentences with visual cues displaying information regarding hand hygiene. This file was developed to provide facts to the participants to aid in the completion of the post-test and to improve baseline knowledge of participants. See Appendix B to visualize the educational PDF file (poster). The post-test contained the same 10 
knowledge questions and three demographic questions, as the pretest. The pre and post-tests was constructed using survey monkey, allowing for analysis of results from both test surveys .

\section{Results}

The pre-test survey was completed by 96 healthcare professionals; two doctors, three nurse practitioners (NPs), 74 registered nurses (RNs) and 17 certified nursing assistants (CNAs). The post-test was completed by 45 healthcare professionals; two doctors, one NP, 37 RNs and five CNAs. There were 60 participants in the pre-test that worked in medical-surgical units and 36 participants that worked in ICUs. There were 28 participants in the post-test that worked in medical-surgical units and 17 participants that worked in ICUs. The total participants from Maryland and Washington, D.C. for the pre-test were 35 and the total for the post-test were 13. The total participants from Vermont for the pre-test were 39 and the total for the post-test were 20. The total participants from Virginia for the pre-test were 22 and the total for the post-test were 13 . The above research has demonstrated an enhancement in hand hygiene knowledge among healthcare providers, one can assume that these individuals will be more motivated to be hand hygiene compliant (Figure 1).

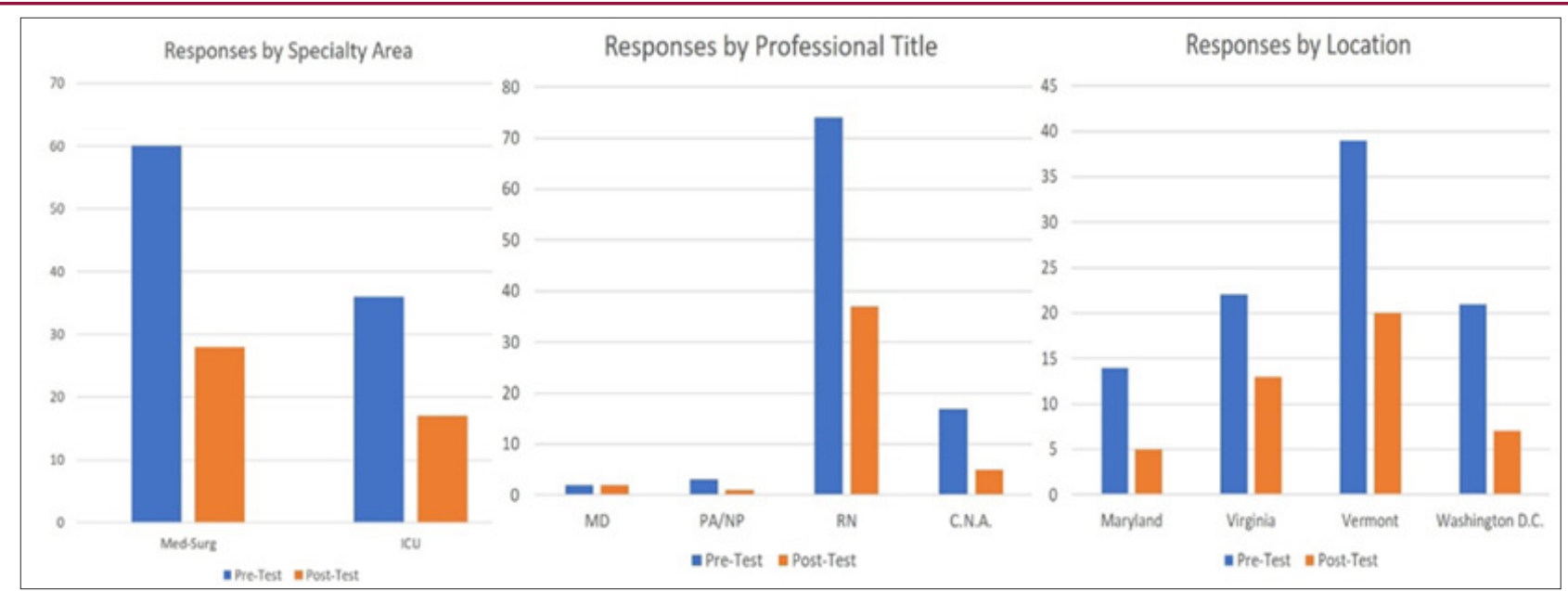

Figure 1: Number of individual participated in test.

\section{Limitations}

There were several limitations noted after analyzing the data. One limitation was there was no way to track whether the same participants completed both the pre-and post-survey. Another limitation includes the fact that when one completed the postsurvey, there was no guarantee that the participant had a secure screen. This means the participant could have had direct reference to the educational materials while completing the post-survey, skewing the results. Finally, another limitation was participant attrition. 96 individuals completed the pre-test, but only 45 individuals completed with post-test (Figure 2).

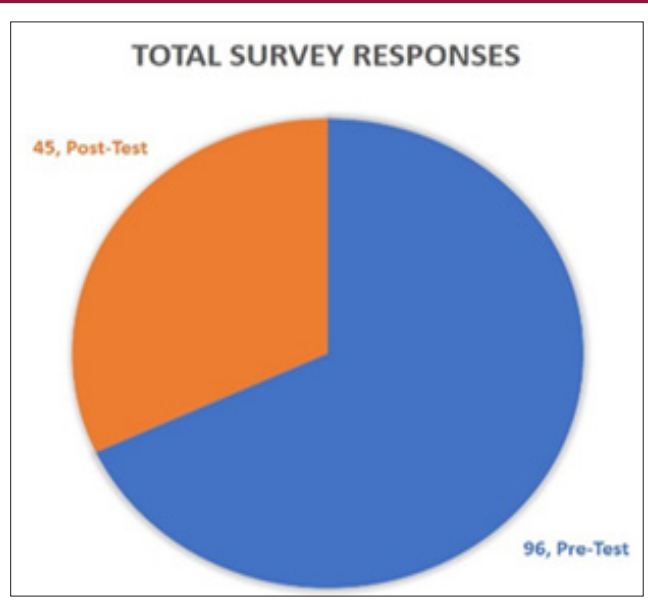

Figure 2: 96 individuals completed pre-test; 45 individuals completed post-test.

\section{Recommendations for Future Research}

After analyzing our data, it has become obvious that there was a lack of physician and midlevel provider participation. To include more of these providers, a different approach to recruit these individuals should be considered. The surveys were sent out via email. Perhaps, most physicians and midlevel providers do not frequently check their email, limiting these individuals from participating. An approach to recruit physicians and midlevel' in future studies might include utilizing text or approaching these providers directly. Furthermore, this research study only analyzed hand hygiene knowledge improvement among healthcare professionals. Therefore, future studies should focus on identifying whether an increase in knowledge results in improved hand hygiene compliance.

\section{Conclusion}

Hand hygiene is an inexpensive and effective way of preventing the spread of infections and in promoting the safety and health of our patients. The purpose of this quality improvement project was to increase knowledge on the importance of hand hygiene and the proper technique of hand hygiene to reduce HAIs. When provided with educational materials on hand hygiene, there was an increase in knowledge among healthcare professionals in acute care settings. After completing a pre-and post-test survey, analyzing hand hygiene knowledge of healthcare professionals, before and after educational material was provided, the data showed an increase in knowledge regarding hand hygiene. The pre-test average score being a $51 \%$ 
and the post-test average score being $75 \%$ shows a significant increase in knowledge regarding importance of hand hygiene. The goal of this project was to raise awareness of the importance of hand hygiene in preventing the spread of nosocomial infections.

\section{References}

1. Center for Disease Control (CDC).

2. World Health Organization (2009) WHO guidelines on hand hygiene in health care: First global patient safety challenge clean care is safer care. Geneva, Sweden: World Health Organization.

3. Haverstick S, Goodrich C, Freeman R, James S, Kullar, et al. (2017) Patients' Hand Washing and Reducing Hospital-Acquired Infection. Critical Care Nurse 37(3): e1-e8.

4. National Institute of Health (NIH).

5. Sadeghi-Moghaddam P, Arjmandnia M, Shokrollahi M, Aghaali M (2015) Does training improve compliance with hand hygiene and decrease infections in the neonatal intensive care unit? A prospective study. Journal of Neonatal-Perinatal Medicine 8(3): 221-225.
6. Nicholas G, K Page, E Martin, David B, Lisa Hall, et al. (2016) CostEffectiveness of a National Initiative to Improve Hand Hygiene Compliance Using the Outcome of Healthcare Associated Staphylococcus aureus Bacteraemia.

7. Kirk J, Kendall A, Marx JF, Pincock T, Young E, et al. (2016) Point of care hand hygiene-where's the rub? A survey of US and Canadian health care workers' knowledge, attitudes, and practices. Am J Infect Control 44(10): 1095-1101.

8. Muller MP, Carter E, Siddiqui N, Larson E (2015) Hand hygiene compliance in an emergency department: The effect of crowding. Academic Emergency Medicine 22(10): 1218-1221.

9. Mark D, Leonard C, Breen H, Graydon R, O'Gorman C, et al. (2014) Mobile phones in clinical practice: Reducing the risk of bacterial contamination. The International Journal of Clinical Practice 68(9): 1060-1064.

10. Nazari R, Seyed Abdolmotaleb H, Khazaeinejad S (2015) Effect of involuntary motivational factors on hand hygiene by health care providers. Journal of Nursing \& Midwifery Sciences 2(4): 24-29.

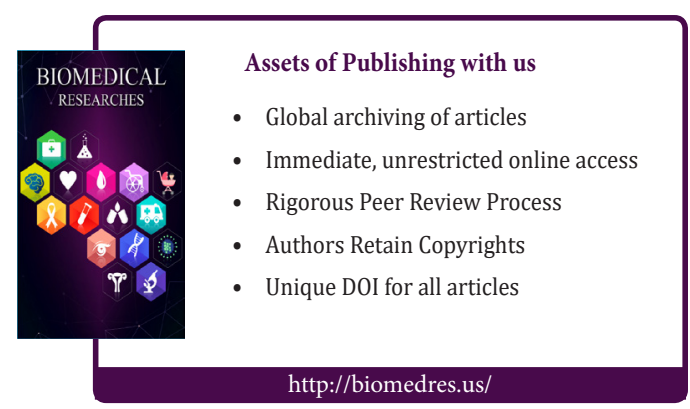

